\title{
Pharmacokinetics of Oseltamivir and Oseltamivir Carboxylate in Critically Ill Patients Receiving Continuous Venovenous Hemodialysis and/or Extracorporeal Membrane Oxygenation
}

Rachel F. Eyler, Pharm.D., Michael Heung, M.D., Melissa Pleva, Pharm.D., Kevin M. Sowinski, Pharm.D., Pauline K. Park, M.D., Lena M. Napolitano, M.D., and Bruce A. Mueller, Pharm.D.

Study Objective. To investigate oseltamivir and oseltamivir carboxylate pharmacokinetics in critically ill patients who were receiving continuous venovenous hemodialysis (CVVHD) and/or extracorporeal membrane oxygenation (ECMO).

Design. Prospective, open-label, pharmacokinetic study.

Setting. Intensive care units of an academic medical center.

Patients. Thirteen critically ill patients aged 13 years or older with suspected or confirmed H1Nl influenza who had a prescription for oseltamivir and were concurrently receiving CVVHD and/or ECMO between October 2009 and January 2010.

Intervention. Oseltamivir $150 \mathrm{mg}$ was administered nasogastrically or nasoenterically every 12 hours. Blood samples were collected at baseline and at $1,2,4,6,8,10$, and 12 hours after administration of the fourth oseltamivir dose or subsequent doses. In patients receiving CVVHD, effluent also was collected at the same time points. Urine was collected throughout the 12-hour dosing interval.

Measurements and Main Results. Eight patients received CVVHD only, four patients received both CVVHD and ECMO, and one patient received ECMO only. Pharmacokinetic parameters for the patient who received only ECMO were not reported. The median maximum plasma concentration and area under the plasma concentration-time curve for the 12-hour dosing interval $\left(\mathrm{AUC}_{0-12}\right)$ for the remaining 12 patients were $83.4 \mathrm{ng} / \mathrm{ml}$ and $216 \mathrm{ng} \cdot$ hour $/ \mathrm{ml}$, respectively, for oseltamivir and $2000 \mathrm{ng} / \mathrm{ml}$ and $21,500 \mathrm{ng} \cdot$ hour/ml, respectively, for oseltamivir carboxylate. Mean clearance due to CVVHD was $33.8 \mathrm{ml} / \mathrm{minute}$ for oseltamivir and $50.2 \mathrm{ml} / \mathrm{min}$ ute for oseltamivir carboxylate. For patients who received ECMO, no substantial differences between pre- and post-ECMO oxygenator plasma concentrations were found for oseltamivir or oseltamivir carboxylate.

Conclusion. Although the optimal pharmacokinetic-pharmacodynamic targets for oseltamivir carboxylate remain unclear, in the patients receiving CVVHD with or without ECMO, a regimen of oseltamivir $150 \mathrm{mg}$ every 12 hours yielded a median oseltamivir carboxylate $\mathrm{AUC}_{0-12}$ considerably higher than would be expected in non-critically ill patients receiving the same dosage regimen. 
Key Words: oseltamivir, oseltamivir carboxylate, critical illness, pharmacokinetics, continuous venovenous hemodialysis, CVVHD, extracorporeal membrane oxygenation, ECMO.

(Pharmacotherapy 2012;32(12):1061-1069)

Oseltamivir, a neuraminidase inhibitor, is indicated for the prophylaxis and treatment of influenza A- and B-related illnesses. During the 2009 influenza A H1N1 pandemic, oseltamivir emerged as a preferred therapy for severe $\mathrm{H} 1 \mathrm{Nl}$ infections. 1,2 In cases of severe illness, the World Health Organization (WHO) recommended higher doses of oseltamivir of up to $150 \mathrm{mg}$ twice/day versus the standard dose of $75 \mathrm{mg}$ twice/day. ${ }^{3}$ However, this recommendation was largely empiric, and the pharmacokinetics of oseltamivir and its active metabolite, oseltamivir carboxylate, had not been investigated thoroughly in critically ill patients receiving continuous renal replacement therapy (CRRT) and/or extracorporeal membrane oxygenation (ECMO). For future influenza pandemics, it will be important to have the disposition of oseltamivir and oseltamivir carboxylate characterized in critically ill patients requiring CRRT and/or ECMO therapies.

Oseltamivir is an oral prodrug that is rapidly converted to oseltamivir carboxylate with approximately $80 \%$ bioavailability in healthy subjects. ${ }^{4}$ Because oseltamivir carboxylate has a low molecular weight of $284.4 \mathrm{Da}$, is less than $3 \%$ protein bound, ${ }^{4}$ and is greater than $99 \%$ renally cleared, it is likely that CRRT would contribute significantly to drug clearance. Furthermore, it is possible that additional mem-

From the College of Pharmacy, University of Michigan, (R.F. Eyler, M. Pleva, and B.A. Mueller), The Renal Replacement Therapy Kinetics Study Group (R.F. Eyler, M. Heung, K.M. Sowinski, and B.A. Mueller), University of Michigan Health System (M. Heung, M. Pleva, P.K. Park, and L.M. Napolitano), and the School of Medicine, University of Michigan (M. Heung, P.K. Park, and L.M. Napolitano), Ann Arbor, Michigan; and the College of Pharmacy, Purdue University, Indianapolis, Indiana (K.M. Sowinski).

Funding was provided by Roche Pharmaceuticals.

The interim analysis of this study was presented in abstract form at the 50th Interscience Conference on Antimicrobial Agents and Chemotherapy meeting, Boston, Massachusetts, September 12-15, 2010.

ClinicalTrials.gov identifier: NCT01048879.

For questions or comments, contact Rachel F. Eyler, Pharm.D., Department of Pharmacy, Yale New Haven Hospital, 55 Park Street, New Haven, CT 06511; e-mail: rachel. eyler@uconn.edu. brane-mediated drug clearance may occur related to ECMO therapy, as has been shown with other drugs. ${ }^{5,6}$ The purpose of this clinical investigation was to describe the pharmacokinetics of oseltamivir and oseltamivir carboxylate in critically ill patients receiving continuous venovenous hemodialysis (CVVHD) and/or ECMO.

\section{Methods}

\section{Study Design and Patient Population}

In this prospective, open-label, pharmacokinetic study, patients aged 13 years or older who were admitted to intensive care units at the University of Michigan (Ann Arbor, MI) with suspected or confirmed H1Nl influenza and who had a prescription for oseltamivir and were receiving CVVHD and/or ECMO were included. Patients were excluded if they had an allergy to oseltamivir, if they were not expected to complete at least 12 hours of CVVHD and/or ECMO therapy, or if it was documented that they were pregnant or breastfeeding.

The institutional review board of the University of Michigan Medical School approved this study. Informed consent was obtained from each patient or a legally authorized guardian before enrollment. Because patients were admitted from other hospitals and often had varied (and sometimes unavailable) previous oseltamivir dosing histories, blood samples were collected with the fourth oseltamivir dose or subsequent doses received at our hospital.

\section{Pharmacologic Treatment}

All patients received oseltamivir $150 \mathrm{mg}$ every 12 hours. Because of their critical illness, none of these patients were able to take drugs by mouth, and the commercial oseltamivir suspension was not available at our institution during the H1N1 pandemic (the study time frame). Consequently, for each dose, the powder from oseltamivir capsules was dissolved in $10-30 \mathrm{ml}$ of water at room temperature and administered by nasogastric or postpyloric feeding tube, followed by a water flush. Oseltamivir phosphate is 
highly water soluble, ${ }^{7}$ and a similar administration technique has been used in other oseltamivir studies in critically ill patients. ${ }^{8}$

\section{Study Procedures}

The CVVHD therapy was delivered using a Prismaflex machine and high-flux polysulfone Prismaflex HF1000 or HF1400 filters with surface areas 1.1 and $1.4 \mathrm{~m}^{2}$, respectively (Gambro, Lakewood, CO). In patients receiving CVVHD, blood samples $(4 \mathrm{ml})$ were obtained from the CVVHD circuit at the sampling port just before the hemodialyzer and were collected into graytopped, sodium fluoride-ethylenediaminetetraacetic acid evacuated blood collection tubes (BD Diagnostic Systems, Franklin Lakes, NJ) at baseline and at $1,2,4,6,8,10$, and 12 hours after the dose was administered. In addition, effluent (spent dialysate plus formed ultrafiltrate) samples $(5 \mathrm{ml})$ were collected at the same time points from the CVVHD circuit into polypropylene cryogenic vials. If the patient received ECMO, blood samples were collected at the same time points from the ECMO circuit directly before and after the oxygenator to quantify drug adsorption to the oxygenator membrane. When patients received both CVVHD and ECMO, CVVHD was performed in parallel with the ECMO circuit, using a separate dedicated dialysis access. Both CVVHD and ECMO sampling procedures were followed, except the ECMO oxygenator samples were limited to the 2- and 12-hour time points. All blood samples were centrifuged, and the plasma was harvested, split, and separated into polypropylene cryogenic vials. Urine was collected for the 12-hour dosing interval in all patients who provided measurable amounts. The total volume was recorded, and a 5-ml aliquot was taken for analysis. Plasma, effluent, and urine samples were stored at $-80^{\circ} \mathrm{C}$ until analysis.

\section{Assays}

All samples were shipped on dry ice to be analyzed at PRA Bioanalytical Laboratory (Assen, the Netherlands). Oseltamivir and oseltamivir carboxylate concentrations in plasma, effluent, and urine samples were determined with liquid chromatography with tandem mass spectrometry. For all three assays, mobile phase A consisted of $0.05 \%$ formic acid in methanol-water (20:80 mixture, volume/volume), and mobile phase B consisted of $0.05 \%$ formic acid in meth- anol-water (95:5 mixture, volume/volume). Tandem mass spectrometric detection was applied using an API 5000 (MDS Sciex, Concord, Canada) mass spectrometer operated in positive ion mode. The data were collected using multiple reaction monitoring. The selected transitions $(\mathrm{m} / \mathrm{z})$ were $313.20 \rightarrow 166.00$ or $313.30 \rightarrow 166.20$ for oseltamivir, $286.20 \rightarrow 138.10$ or $286.30 \rightarrow 138.10$ for oseltamivir carboxylate, $316.20 \rightarrow 167.20$ or $316.30 \rightarrow 167.20$ for oseltamivir RO0640796-003002 (deuterated internal standard for oseltamivir), and $288.20 \rightarrow 139.10$ or $288.30 \rightarrow 139.10$ for R00640802-004-002 (deuterated internal standard for oseltamivir carboxylate).

The lower limits of detection for oseltamivir in plasma, effluent, and urine samples were 1, 0.5 , and $5 \mathrm{ng} / \mathrm{ml}$, respectively. The lower limits of detection for oseltamivir carboxylate were 10 , 5 , and $30 \mathrm{ng} / \mathrm{ml}$, respectively. Coefficients of variation were $3.2-8.0 \%$ for oseltamivir and 3.4$7.4 \%$ for oseltamivir carboxylate plasma concentrations, $1.4-4.5 \%$ for oseltamivir and $1.1-2.4 \%$ for oseltamivir carboxylate effluent concentrations, and $1.1-3.9 \%$ for oseltamivir and 1.1$3.1 \%$ for oseltamivir carboxylate urine concentrations.

\section{Pharmacokinetic Analysis}

Patients treated with CVVHD received a prefilter citrate infusion for regional anticoagulation. $^{9}$ Because of this, plasma concentrations were adjusted to account for plasma dilution due to the citrate infusion. ${ }^{10}$ Pharmacokinetic parameter estimation was performed using noncompartmental methods. The maximum plasma concentration during the dosing interval $\left(\mathrm{C}_{\max }\right)$ was determined by observation of the plasma concentration data. The elimination constant $(\mathrm{k})$ was calculated by least squares linear regression of the log-linear portion of the plasma concentration-time curve. Terminal elimination half-life was calculated as $0.693 / \mathrm{k}$. The area under the curve for the 12-hour dosing interval $\left(\mathrm{AUC}_{0-12}\right)$ and the area under the moment of the plasma concentration-time curve were calculated using the linear trapezoidal rule. In two cases when data points were not available at 12 hours, they were extrapolated from the decay curve to 12 hours to allow the estimation of $\mathrm{AUC}_{0-12}$. Mean residence time at steady state $\left(\mathrm{MRT}_{\mathrm{ss}}\right)$ was calculated as the area under the moment of the plasma concentration-time curve for the dosing period/AUC ${ }_{0-12}{ }^{11}$ Total clearance $\left(\mathrm{Cl}_{\mathrm{T}}\right)$, defined as $\mathrm{Cl}_{\mathrm{NR}}+\mathrm{Cl}_{\mathrm{R}}+\mathrm{Cl}_{\mathrm{CVVHD}}$, where $\mathrm{Cl}_{\mathrm{NR}}, \mathrm{Cl}_{\mathrm{R}}$, and 
$\mathrm{Cl}_{\mathrm{CVVHD}}$ are nonrenal, renal, and CVVHD drug clearance, respectively, was calculated as dose/ $\mathrm{AUC}_{0-12}$. The steady-state volume of distribution $\left(\mathrm{V}_{\mathrm{ss}}\right)$ was calculated as MRT $\cdot \mathrm{Cl}_{\mathrm{T}} \cdot{ }^{12}$ Because the dose administered was an oral dose, and bioavailability $(\mathrm{F})$ could not be calculated, $\mathrm{Cl}_{\mathrm{T}}$ and $\mathrm{V}_{\mathrm{ss}}$ for oseltamivir are reported as $\mathrm{Cl}_{\mathrm{T}} / \mathrm{F}$ and $\mathrm{V}_{\text {ss }} / \mathrm{F}$.

The saturation coefficient (SA) for oseltamivir and oseltamivir carboxylate at each time point was calculated as effluent concentration/prehemodialysis filter plasma concentration. The $\mathrm{Cl}_{\mathrm{CVVHD}}$ was calculated as effluent rate - SA. The $\mathrm{Cl}_{\mathrm{R}}$ was calculated as (urine drug concentration - total urine volume)/AUC $\mathrm{A}-12 .^{13}$ Creatinine clearance was calculated as (urine creatinine concentration - urine volume)/(serum creatinine concentration $-12 \mathrm{hrs}$ ). Pearson correlation coefficients were calculated to examine the relationship between $\mathrm{Cl}_{R}$ and creatinine clearance. Linear regressions were used to test the effect of weight or body mass index (BMI) on pharmacokinetic parameters. A $\mathrm{p}$ value of less than 0.05 indicated a statistically significant difference when examining the effects of weight or BMI and administration route on $\mathrm{AUC}_{0-12}$.

\section{Results}

Between October 2009 and January 2010, 14 patients were enrolled in the study. Nine patients received CVVHD only, four received
CVVHD in addition to ECMO, and one patient received ECMO only. One patient discontinued CVVHD at 2.5 hours due to clinical improvement and was excluded from the final analysis. Demographic characteristics of the 13 patients who completed the trial are presented in Table 1. Pharmacokinetic parameters for the patient who received only ECMO were not reported because the data set did not allow for the calculation of key pharmacokinetic parameters. No adverse effects related to oseltamivir treatment were noted in any of the patients during the 12-hour study interval. Five (38\%) of the 13 patients were receiving therapy with vasopressors (norepinephrine, phenylephrine, or vasopressin) during the sampling interval. Seven (54\%) of the 13 patients survived until transfer or discharge from the intensive care unit.

Figure 1 illustrates the oseltamivir and oseltamivir carboxylate plasma concentration-time curves for the 12 patients who received CVVHD and illustrates the intersubject variability of oseltamivir and oseltamivir carboxylate. Median values and interquartile ranges (IQRs) from the pharmacokinetic analysis are shown in Table 2. The median (IQR) AUC $0-12$ was $216 \mathrm{ng} \bullet$ hour $/ \mathrm{ml}$ (156-317 $\mathrm{ng} \bullet \mathrm{hr} / \mathrm{ml})$ for oseltamivir, and the median (IQR) $\mathrm{AUC}_{0-12}$ was 21,500 ng $\bullet$ hour/ml $(13,300-34,400 \mathrm{ng} \bullet \mathrm{hr} / \mathrm{ml})$ for oseltamivir carboxylate for the 12 patients. The median (IQR) oseltamivir carboxylate concentration at the end

Table 1. Baseline Demographics of the 13 Study Patients

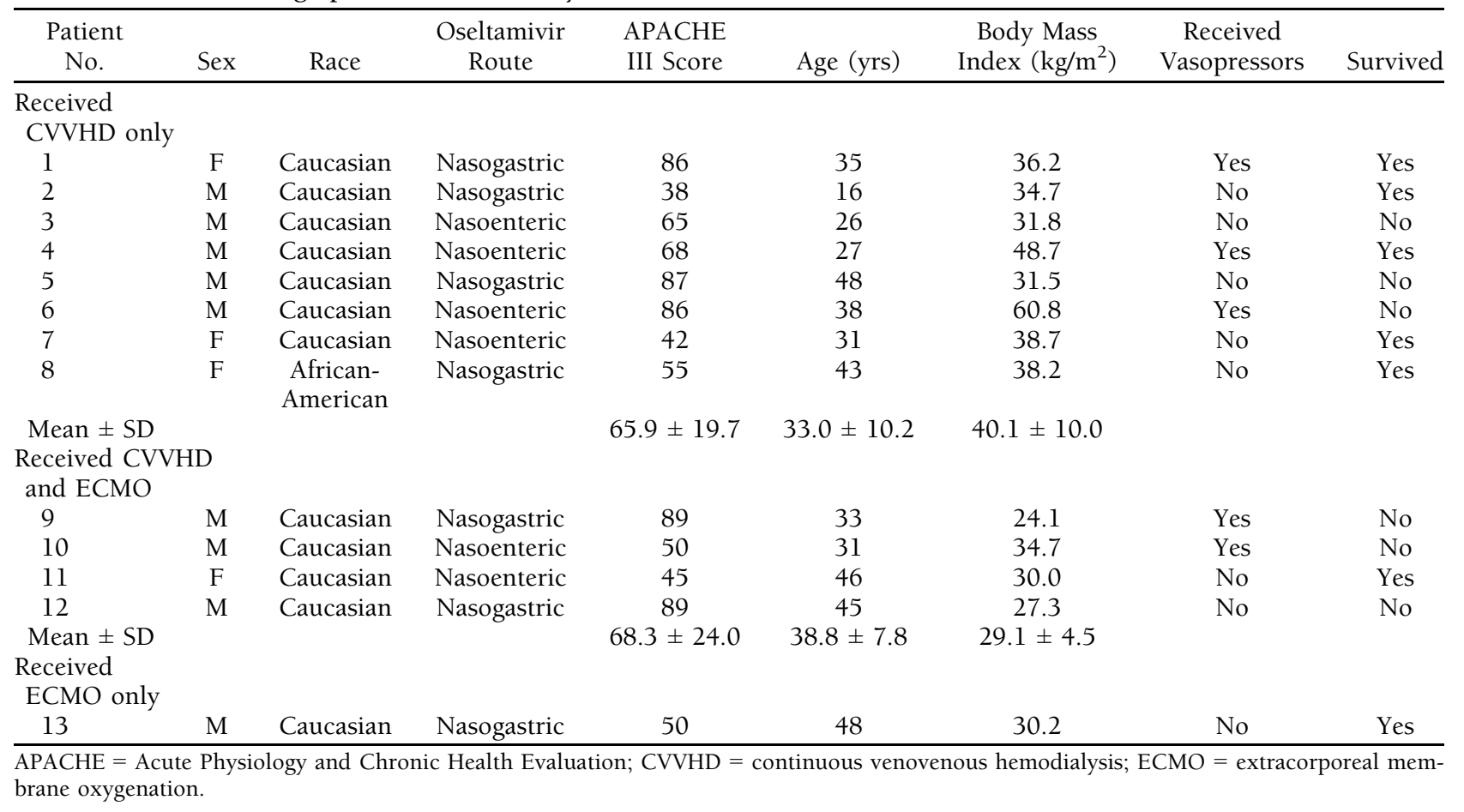


a)

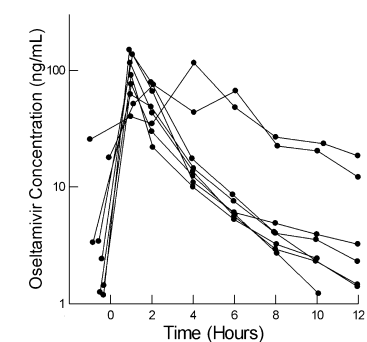

c)

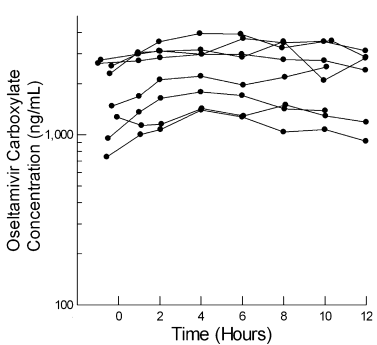

b)
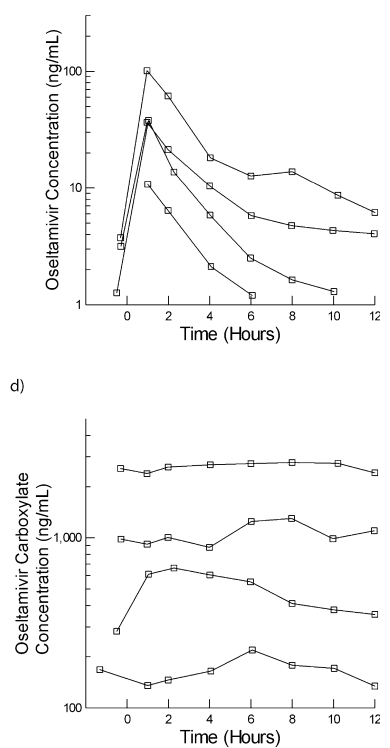

Figure 1. Oseltamivir and oseltamivir carboxylate plasma concentrations versus time over the 12-hour dosing interval in the 12 patients who received continuous venovenous hemodialysis (CVVHD). Closed circles represent patients receiving CVVHD only, and open squares represent patients who received both CVVHD and extracorporeal membrane oxygenation. Lines connecting the data points are for clarity and were not modeled.

of the dosing interval (12 hrs) was $1760 \mathrm{ng} / \mathrm{ml}$ (1050-2720 ng/ml).

No significant correlation between weight and $\mathrm{AUC}_{0-12}$ was noted for either oseltamivir or oseltamivir carboxylate. In addition, no significant correlation between BMI and the aforementioned pharmacokinetic parameters was noted; however, patients receiving ECMO had substantially smaller BMIs than patients receiving CVVHD only (Table 1).

In the 12 patients who received CVVHD, the mean \pm SD blood, dialysate, and effluent flow rates were $196 \pm 14.4,2420 \pm 764$, and $3300 \pm$ $919 \mathrm{ml} /$ hour $(30.8 \pm 3.57 \mathrm{ml} / \mathrm{kg} / \mathrm{hr})$, respectively. Saturation coefficients were $0.62 \pm 0.11$ for oseltamivir and $0.94 \pm 0.11$ for oseltamivir carboxylate. Mean $\mathrm{Cl}_{\mathrm{CVvHD}}$ values were $33.8 \pm$ $9.4 \mathrm{ml} /$ minute for oseltamivir and $50.2 \pm 7.0 \mathrm{ml} /$ minute for oseltamivir carboxylate. For the four patients who received ECMO, pre- and postECMO oxygenator oseltamivir and oseltamivir carboxylate concentrations are presented in Table 3.

Urine samples were collected for 10 of the 13 patients. Of the three patients for whom urine samples were not available, two did not have a urinary catheter in place because the patients were anuric, and one patient's urine collection was emptied inadvertently before the 12-hour interval was completed. The amount of oseltamivir (parent or active metabolite) appearing in the urine was minimal for most patients. A significant correlation was noted between oseltamivir $\mathrm{Cl}_{R}$ and calculated creatinine clearance $(\mathrm{r}=0.91, \mathrm{p}<0.001)$ as well as oseltamivir carboxylate $\mathrm{Cl}_{\mathrm{R}}$ and calculated creatinine clearance $(\mathrm{r}=0.88, \mathrm{p}=0.002)$. The median (IQR) values for oseltamivir $\mathrm{Cl}_{R}$ and oseltamivir carboxylate $\mathrm{Cl}_{R}$ were $22.53 \mathrm{ml} /$ minute $(14.1-52.95 \mathrm{ml} / \mathrm{min})$ and $1.86 \mathrm{ml} /$ minute $(0.91-10.83 \mathrm{ml} / \mathrm{min})$, respectively.

Table 2. Pharmacokinetic Parameters of Oseltamivir and Oseltamivir Carboxylate in the 12 Study Patients ${ }^{\mathrm{a}}$

\begin{tabular}{|c|c|c|}
\hline Parameter & $\begin{array}{l}\text { CVVHD-Only Group } \\
(\mathrm{n}=8)\end{array}$ & $\begin{array}{l}\text { CVVHD and ECMO Group } \\
(\mathrm{n}=4)\end{array}$ \\
\hline \multicolumn{3}{|l|}{ Oseltamivir } \\
\hline $\mathrm{C}_{\max }(\mathrm{ng} / \mathrm{ml})$ & $103(75.9-121)$ & $37.3(30.1-53.8)$ \\
\hline $\mathrm{C}_{12}(\mathrm{ng} / \mathrm{ml})$ & $2.25(1.48-5.45)$ & $2280(1310-2840)$ \\
\hline $\mathrm{T}_{\max }(\mathrm{hrs})$ & $1.0(1.0-1.3)$ & $1.0(1.0-1.0)$ \\
\hline $\mathrm{Vd}_{\mathrm{ss}} / \mathrm{F}(\mathrm{L} / \mathrm{F})$ & $1460(1330-2200)$ & $4500(3760-6990)$ \\
\hline $\mathrm{Cl}_{\mathrm{T}} / \mathrm{F}(\mathrm{L} / \mathrm{hr} / \mathrm{F})$ & $578(414-787)$ & $1470(1020-2570)$ \\
\hline Half-life (hrs) & $4.1(3.0-6.8)$ & $4.8(3.8-8.4)$ \\
\hline $\mathrm{AUC}_{0-12}(\mathrm{ng} \cdot \mathrm{hr} / \mathrm{ml})$ & $263(192-373)$ & $106(72.5-171)$ \\
\hline \multicolumn{3}{|c|}{ Oseltamivir carboxylate } \\
\hline $\mathrm{C}_{\max }(\mathrm{ng} / \mathrm{ml})$ & $2670(1710-3580)$ & $981(553-1670)$ \\
\hline $\mathrm{C}_{12}(\mathrm{ng} / \mathrm{ml})$ & $2.50(0.725-4.63)$ & $727(300-1430)$ \\
\hline $\mathrm{T}_{\max }(\mathrm{hrs})$ & $4.0(4.0-6.5)$ & $7.0(5.1-8.0)$ \\
\hline Half-life (hrs) & $22.3(19.1-33.6)$ & $14.4(10.1-19.3)$ \\
\hline $\mathrm{AUC}_{0-12}(\mathrm{ng} \bullet \mathrm{hr} / \mathrm{ml})$ & $29,500(17,600-35,800)$ & $9390(5000-17,600)$ \\
\hline
\end{tabular}

Data are median (interquartile range).

CVVHD = continuous venovenous hemodialysis; $\mathrm{ECMO}=$ extracorporeal membrane oxygenation; $\mathrm{C}_{\max }=$ maximum concentration during the dosing interval; $\mathrm{C}_{12}=$ concentration at the end of the 12-hr dosing interval (trough); $\mathrm{T}_{\max }=$ time of maximum concentration; $\mathrm{Vd} \mathrm{ss}_{\mathrm{ss}}=\mathrm{vol}_{-}$ ume of distribution at steady state; $\mathrm{F}=$ bioavailability; $\mathrm{Cl}_{\mathrm{T}}=$ total clearance; $\mathrm{AUC}_{0-12}=$ area under the plasma concentration-time curve from $0-12$ hrs.

${ }^{\text {a }}$ Pharmacokinetic parameters for the patient who received only ECMO were not reported. 
Table 3. Oseltamivir and Oseltamivir Carboxylate Plasma Concentrations Before and After the ECMO Oxygenator

\begin{tabular}{|c|c|c|c|c|c|c|}
\hline \multirow[b]{2}{*}{ Patient No. } & \multirow{2}{*}{$\begin{array}{l}\text { No. of days } \\
\text { ECMO Received } \\
\text { Before the Study }\end{array}$} & \multirow[b]{2}{*}{$\begin{array}{l}\text { Time After Oseltamivir } \\
\text { Dose Administered (hrs) }\end{array}$} & \multicolumn{2}{|c|}{$\begin{array}{c}\text { Oseltamivir } \\
\text { Concentration } \\
(\mathrm{ng} / \mathrm{ml})\end{array}$} & \multicolumn{2}{|c|}{$\begin{array}{c}\text { Oseltamivir Carboxylate } \\
\text { Concentration } \\
(\mathrm{ng} / \mathrm{ml})\end{array}$} \\
\hline & & & $\begin{array}{c}\text { Before } \\
\text { Oxygenator }\end{array}$ & $\begin{array}{c}\text { After } \\
\text { Oxygenator }\end{array}$ & $\begin{array}{c}\text { Before } \\
\text { Oxygenator }\end{array}$ & $\begin{array}{c}\text { After } \\
\text { Oxygenator }\end{array}$ \\
\hline \multicolumn{7}{|l|}{$\begin{array}{l}\text { CVVHD } \\
\text { and ECMO }\end{array}$} \\
\hline \multirow[t]{2}{*}{9} & 7 & 2 & 8.73 & 5.92 & 169 & 180 \\
\hline & & 12 & BLLQ & BLLQ & 145 & 200 \\
\hline \multirow[t]{2}{*}{10} & 7 & 2 & 13.3 & 12.0 & 656 & 726 \\
\hline & & 12 & 73.3 & 62.5 & 351 & 364 \\
\hline \multirow[t]{2}{*}{11} & 5 & 2 & 22.1 & 20.6 & 987 & 923 \\
\hline & & 12 & 5.5 & 5.7 & 731 & 964 \\
\hline \multirow[t]{2}{*}{12} & 2 & 2 & 65.1 & 57.6 & 2860 & 2740 \\
\hline & & 12 & 6.9 & 7.0 & 2460 & 2570 \\
\hline \multicolumn{7}{|l|}{ ECMO only } \\
\hline \multirow[t]{7}{*}{13} & 2 & 1 & 9.3 & 10.5 & 936 & 967 \\
\hline & & 2 & 11.1 & 11.2 & 894 & 967 \\
\hline & & 4 & 10.6 & 10.6 & 908 & 975 \\
\hline & & 6 & 13.4 & 12.3 & 865 & 887 \\
\hline & & 8 & 5.7 & 7.3 & 876 & 853 \\
\hline & & 10 & 7.0 & 7.9 & 1000 & 896 \\
\hline & & 12 & 13.2 & 14.0 & 913 & 1050 \\
\hline
\end{tabular}

ECMO = extracorporeal membrane oxygenation; BLLQ = below the lower limit of quantification.

\section{Discussion}

This prospective trial was designed to characterize oseltamivir and oseltamivir carboxylate pharmacokinetics in patients receiving CVVHD and/or ECMO. For the 12 patients who received CVVHD, the mean saturation coefficients of $0.62 \pm 0.11$ for oseltamivir and $0.94 \pm 0.11$ for oseltamivir carboxylate correlate well with the respective plasma protein binding values of $42 \%$ and 3\% reported in healthy volunteers. ${ }^{4}$ Oseltamivir carboxylate freely crossed the CVVHD membrane, and $\mathrm{Cl}_{\mathrm{CVVHD}}$ was an important route of elimination in these patients. In the five patients receiving ECMO, pre- and postoxygenator membrane concentrations of oseltamivir and oseltamivir carboxylate did not differ substantially (Table 3 ), suggesting that drug binding to the oxygenator was not a clinically relevant source of drug clearance.

In this trial, oseltamivir and oseltamivir carboxylate pharmacokinetic parameters exhibited striking variability. The lowest and highest $C_{\max }$ differed by 14-fold for oseltamivir and 18-fold for oseltamivir carboxylate. Although a substantial portion of the intersubject variability in oseltamivir carboxylate concentrations may be due to differences in the number of previous oseltamivir doses, this study also agrees with previous findings that pharmacokinetic variability can be increased in critical illness and multiorgan failure, ${ }^{14}$ particularly when an oral drug is administered. ${ }^{15,16}$

Many factors may have contributed to the pharmacokinetic variability seen in these critically ill patients. ${ }^{14}, 17,18$ Administration route (nasogastric vs nasoenteric) may have contributed to variability, although there were no significant differences in oseltamivir and oseltamivir carboxylate $\mathrm{AUC}_{0-12}$ between groups receiving different routes of administration. It is also possible that oseltamivir could have partially bound to the nasogastric or nasoenteric tubing. Oseltamivir was administered without regard to enteral feedings, which all but two patients (patient nos. 6 and 10) were receiving. The bioavailability of oseltamivir with enteral feedings has not been studied, although coadministration of oseltamivir with food does not significantly affect $\mathrm{AUC}_{\mathrm{O}-12}{ }^{19} \mathrm{~A}$ study of the pharmacokinetics of oseltamivir in critically ill patients found similar median plasma concentrations in patients receiving and those not receiving enteral feedings. ${ }^{8}$

Another contributor to the pharmacokinetic variability seen in our patients could be due to variations in the expression and activity of human carboxylesterase 1 , the hepatic enzyme responsible for the hydrolysis of oseltamivir to oseltamivir carboxylate. Expression of human carboxylesterase 1 messenger RNA has been reported to vary by a factor of 12 in adults, ${ }^{20}$ 
and genetic polymorphism, proinflammatory cytokine secretion, ${ }^{21}$ and drug-drug interactions $^{22}$ may impair formation of the active metabolite. However, clinically relevant inhibition of oseltamivir conversion leading to accumulation of the parent compound is unlikely, as the oseltamivir carboxylate concentrations were consistently higher than the oseltamivir concentrations for all patients (the oseltamivir carboxylate AUC:oseltamivir AUC ratio ranged from 32-172) (Table 4).

In our patients, variations in oseltamivir and oseltamivir carboxylate concentrations were unlikely to be due to drug-drug interactions because most patients were not receiving potentially interacting drugs. In vitro trials have reported carboxylesterase 1 inhibition with tamoxifen, thioridazine, aripiprazole, perphenazine, fluoxetine, and certain statin drugs, 23,24 and it is possible that oseltamivir hydrolysis may be competitively inhibited by other carboxylesterase 1 substrates, such as clopidogrel, certain angiotensin-converting enzyme inhibitors, meperidine, and methylphenidate. $22,23,25,26$ The only patient to receive any of these potentially interacting drugs was patient no. 10, who was receiving fluoxetine. This patient had the second lowest parent oseltamivir concentrations of all patients, suggesting that limited conversion to oseltamivir carboxylate was not a clinically significant factor in this patient. In addition, no patients were receiving probenecid, a drug found to inhibit the tubular excretion of oseltamivir carboxylate. $^{27}$

It is not surprising that given the prolonged half-lives of oseltamivir carboxylate in our patients (22. $3 \mathrm{hrs}$ in patients receiving CVVHD only and $14.4 \mathrm{hrs}$ in patients receiving ECMO and CVVHD), oseltamivir carboxylate exposure in our study was substantially higher compared with that expected in healthy volunteers receiving the same dose. ${ }^{28}$ Median oseltamivir carboxylate $\mathrm{AUC}_{0-12}$ values reported in our study were elevated considerably compared with those reported in a multidose study of healthy volunteers $(21,500$ vs $4904 \mathrm{ng} \cdot \mathrm{hr} / \mathrm{ml}),{ }^{28}$ suggesting that a dosage reduction may be warranted from the dosage of $150 \mathrm{mg}$ every 12 hours used in our study.

Oseltamivir carboxylate exposure was decreased compared with another report of oseltamivir carboxylate pharmacokinetics in critically ill adults requiring CRRT. ${ }^{8}$ That study evaluated the pharmacokinetics of oseltamivir and oseltamivir carboxylate in five critically ill adults who received oseltamivir at standard dosages of $75 \mathrm{mg}$ twice/day. The median oseltamivir carboxylate $\mathrm{AUC}_{0-12}$ reported for the five patients $(28,023 \mathrm{ng} \cdot \mathrm{hr} / \mathrm{ml})$, when doubled to account for the difference in dosage regimens, was substantially higher than that found in our eight patients who received CVVHD without ECMO $(29,500 \mathrm{ng} \bullet \mathrm{hr} / \mathrm{ml})$. Although all patients in both studies had received at least three doses of oseltamivir before pharmacokinetic sampling occurred, oseltamivir dosing histories were varied, which may account for some of the difference. However, the mode of CRRT, effluent rate used, and degree of residual renal function were not reported in the other study, ${ }^{8}$ making a direct comparison with our study difficult. It may be that some of the large difference in oseltamivir carboxylate exposure may be attributed to increased $\mathrm{Cl}_{\mathrm{T}}$ due to higher CRRT effluent flow rates (as well as variations in CRRT mode, filter type, etc.) or greater residual $\mathrm{Cl}_{\mathrm{R}}$ values in our patients.

Table 4. Oseltamivir Carboxylate AUC:Oseltamivir AUC Ratios

\begin{tabular}{cccc}
\hline Patient No. & $\begin{array}{c}\text { Oseltamivir } \\
\text { AUC (ng•hr/ml) }\end{array}$ & $\begin{array}{c}\text { Oseltamivir } \\
\text { Carboxylate AUC (ng•hr/ml) }\end{array}$ & $\begin{array}{c}\text { Oseltamivir Carboxylate } \\
\text { AUC:Oseltamivir AUC Ratio }\end{array}$ \\
\hline 1 & 234 & 34,900 & 149 \\
2 & 492 & 15,700 & 32 \\
3 & 167 & 24,800 & 149 \\
4 & 170 & 18,200 & 107 \\
5 & 333 & 41,900 & 126 \\
6 & 199 & 34,300 & 172 \\
7 & 292 & 13,400 & 46 \\
8 & 553 & 38,600 & 70 \\
9 & 30 & 2050 & 68 \\
10 & 87 & 5990 & 69 \\
11 & 125 & 12,800 & 102 \\
Median (IQR) & 311 & 32,200 & 104 \\
\hline
\end{tabular}

$\mathrm{AUC}=$ area under the plasma concentration-time curve; IQR = interquartile range. 
Unlike the previous report, ${ }^{8}$ our study included patients receiving ECMO. It is worthwhile to note that although pre- and post-ECMO oxygenator concentrations did not vary considerably, the median oseltamivir carboxylate $\mathrm{AUC}_{0-12}$ for patients receiving both CVVHD and ECMO $(9390 \mathrm{ng} / \mathrm{ml} \cdot \mathrm{hr})$ was considerably lower than that achieved in patients receiving only CVVHD $(29,500 \mathrm{ng} / \mathrm{ml} \cdot \mathrm{hr})$. An increased volume of distribution may have contributed to the decreased oseltamivir carboxylate exposure as well (4500 L/F in the CVVHD and ECMO group vs $1460 \mathrm{~L} / \mathrm{F}$ in the CVVHD-only group). Although patients receiving ECMO often receive large volumes of blood products that lead to increases in volume of distribution, this may not fully explain the more than 3-fold difference in $\mathrm{V}_{\mathrm{ss}} / \mathrm{F}$ that was observed. Another possible explanation of the pharmacokinetic variability could be that oseltamivir exhibited decreased bioavailability in patients receiving CVVHD and ECMO. Our ECMO study population was too small to draw firm conclusions about oseltamivir bioavailability. However, an instance of suspected poor oral oseltamivir bioavailability in a 14-year-old patient with suspected gastric dysfunction receiving CRRT and ECMO has been reported in a case series, ${ }^{29}$ although the other two patients in this same series, as well as a patient in a different case report, ${ }^{30}$ have exhibited much higher oseltamivir carboxylate concentrations.

\section{Limitations}

An important limitation of this study is that previous oseltamivir dosing history in our patients varied. Consequently, some of the pharmacokinetic variability seen with oseltamivir carboxylate could have been due to differences in the number of previous doses received. Because oseltamivir was administered twice/day, sampling in our trial was limited to a single 12-hour dosing interval. Oseltamivir carboxylate often exhibited a half-life longer than the dosing interval itself, making the half-life difficult to characterize. The oral dosage form was not radiolabeled, preventing us from acquiring information about oral bioavailability. Consequently, volume of distribution and $\mathrm{Cl}_{\mathrm{T}}$ calculations were affected by oral bioavailability, which is a source of variability in critically ill patients. ${ }^{16}$ Finally, although this study investigated the pharmacokinetics of oseltamivir in critically ill patients receiving CVVHD and/or ECMO, it did not investigate viral load or clinical outcomes.
Despite these limitations, it remains clear that the median oseltamivir carboxylate $\mathrm{AUC}_{0-12}$ was substantially higher than would be expected in non-critically ill patients administered the same oseltamivir dose.

Oseltamivir Carboxylate Pharmacodynamics and Dosing Implications

One study reported a mean \pm SD oseltamivir carboxylate $50 \%$ inhibitory concentration $\left(\mathrm{IC}_{50}\right)$ of $0.186 \pm 0.107 \mathrm{ng} / \mathrm{ml}$ for the $2009 \mathrm{H} 1 \mathrm{Nl}$ influenza pandemic. ${ }^{31}$ The median 12-hour oseltamivir carboxylate concentration of $1760 \mathrm{ng} / \mathrm{ml}$ found in our study was more than 9000-fold higher than this reported $\mathrm{IC}_{50}$, suggesting that a decrease in dosage is indeed indicated in these patients. The magnitude of the optimal dosage decrease is less clear. Although conventional oseltamivir dosing appears to yield sufficient drug exposure to treat influenza infections, the optimal oseltamivir carboxylate $\mathrm{AUC}_{0-24}$ value has yet to be derived. Furthermore, human data correlating oseltamivir carboxylate concentrations in the plasma with concentrations in the alveolar fluid have not been established, ${ }^{32}$ and this correlation should be clarified to optimize dosing. These issues, coupled with the large pharmacokinetic variability seen in our study, make dosing recommendations difficult to make in this population.

Although achieving high oseltamivir plasma concentrations may lead to adverse effects (e.g., nausea, vomiting, neurologic effects), ${ }^{32}$ oseltamivir is well tolerated, and these risks need to be balanced against the variability of pharmacokinetics found in this severely ill population. The package insert recommends a dosage of $75 \mathrm{mg}$ once/day for patients with reduced creatinine clearances of $10-30 \mathrm{ml} /$ minute, ${ }^{19}$ and this recommended dosage seems reasonable to apply to critically ill patients requiring CVVHD at the effluent rates used in this trial. Centers using CRRT at higher effluent rates may require more aggressive dosing. Because the patients in our study who received ECMO tended to have lower AUC values, a higher dose might be considered in these patients, particularly in cases where gastrointestinal dysfunction is suspected. ${ }^{29}$

\section{Conclusion}

In patients receiving CVVHD, oseltamivir carboxylate freely crosses the hemodialysis filter, and oseltamivir carboxylate clearance from CVVHD represents an important route of elimi- 
nation in these patients. Use of ECMO does not appear to contribute substantially to oseltamivir carboxylate clearance. The oseltamivir carboxylate $\mathrm{AUC}_{0-12}$ in critically ill patients receiving CVVHD with or without ECMO was considerably higher than those reported in healthy volunteers. In our critically ill patients receiving CVVHD, a regimen of oseltamivir $150 \mathrm{mg}$ every 12 hours produced a median oseltamivir carboxylate $\mathrm{AUC}_{0-12}$ substantially higher than would be expected in non-critically ill patients receiving the same oseltamivir regimen.

\section{References}

1. Jain S, Kamimoto L, Bramley AM, et al. Hospitalized patients with 2009 HlNl influenza in the United States, April-June 2009. N Engl J Med 2009;361:1935-44.

2. Smith JR, Ariano RE, Toovey S. The use of antiviral agents for the management of severe influenza. Crit Care Med 2010;38 (suppl):e43-51.

3. World Health Organization. WHO guidelines for clinical management of human infection with pandemic (HINl) 2009; revised guidance, 2009. Available from http://www.who.int/csr/ resources/publications/swineflu/clinical_management/en/index. htmL. Accessed January 18, 2011.

4. He G, Massarella J, Ward P. Clinical pharmacokinetics of the prodrug oseltamivir and its active metabolite Ro 64-0802. Clin Pharmacokinet 1999;37:471-84.

5. Mulla H, Pooboni S. Population pharmacokinetics of vancomycin in patients receiving extracorporeal membrane oxygenation. Br J Clin Pharmacol 2005;60:265-75.

6. Spriet I, Annaert P, Meersseman P, et al. Pharmacokinetics of caspofungin and voriconazole in critically ill patients during extracorporeal membrane oxygenation. J Antimicrob Chemother 2009;63:767-70.

7. Green MD, Nettey H, Wirtz RA. Determination of oseltamivir quality by colorimetric and liquid chromatographic methods. Emerg Infect Dis 2008;14:552-6.

8. Ariano RE, Sitar DS, Zelenitsky SA, et al. Enteric absorption and pharmacokinetics of oseltamivir in critically ill patients with pandemic (H1N1) influenza. CMAJ 2010;182:357-63.

9. Swartz R, Pasko D, O'Toole J, Starmann B. Improving the delivery of continuous renal replacement therapy using regional citrate anticoagulation. Clin Nephrol 2004;61:134-43.

10. Vilay AM, Grio M, DePestel DD, et al. Daptomycin pharmacokinetics in critically ill patients receiving continuous venovenous hemodialysis. Crit Care Med 2011;39:19-25.

11. Gibaldi M, Perrier D. Pharmacokinetics, 2nd ed. New York: Marcel Dekker, Inc., 1982.

12. Benet LZ, Galeazzi RL. Noncompartmental determination of the steady-state volume of distribution. J Pharm Sci 1979;68:1071-4.

13. Gajjar DA, Bello A, Ge Z, Christopher L, Grasela DM. Multipledose safety and pharmacokinetics of oral garenoxacin in healthy subjects. Antimicrob Agents Chemother 2003;47:2256-63.

14. Roberts DM, Roberts JA, Roberts MS, et al. Variability of antibiotic concentrations in critically ill patients receiving continuous renal replacement therapy: a multicentre pharmacokinetic study. Crit Care Med 2012;40:1523-8.

15. Barquist ES, Gomez-Fein E, Block EF, Collin G, Alzamel H, Martinez O. Bioavailability of oral fluconazole in critically ill abdominal trauma patients with and without abdominal wall closure: a randomized crossover clinical trial. J Trauma 2007;63:159-63.

16. Kruger PS, Freir NM, Venkatesh B, Robertson TA, Roberts MS, Jones M. A preliminary study of atorvastatin plasma concentrations in critically ill patients with sepsis. Intensive Care Med 2009;35:717-21.

17. Eyler RF, Mueller BA. Antibiotic dosing in critically ill patients with acute kidney injury. Nat Rev Nephrol 2011;7:226-35.

18. Shekar K, Fraser JF, Smith MT, Roberts JA. Pharmacokinetic changes in patients receiving extracorporeal membrane oxygenation. [published online ahead of print April 18, 2012]. J Crit Care. Available from http://www.jccjournal.org/article/ S0883-9441\%2812\%2900092-5/abstract.

19. Genentech USA, Inc. Tamiflu (oseltamivir) prescribing information. South San Francisco, CA; 2011.

20. Yang D, Pearce RE, Wang X, Gaedigk R, Wan YJ, Yan B. Human carboxylesterases HCE1 and HCE2: ontogenic expression, inter-individual variability and differential hydrolysis of oseltamivir, aspirin, deltamethrin and permethrin. Biochem Pharmacol 2009;77:238-47.

21. Yang J, Shi D, Yang D, Song X, Yan B. Interleukin-6 alters the cellular responsiveness to clopidogrel, irinotecan, and oseltamivir by suppressing the expression of carboxylesterases HCE1 and HCE2. Mol Pharmacol 2007;72:686-94.

22. Shi D, Yang J, Yang D, et al. Anti-influenza prodrug oseltamivir is activated by carboxylesterase human carboxylesterase 1 , and the activation is inhibited by antiplatelet agent clopidogrel. J Pharmacol Exp Ther 2006;319:1477-84.

23. Zhu HJ, Appel DI, Peterson YK, Wang Z, Markowitz JS. Identification of selected therapeutic agents as inhibitors of carboxylesterase 1: potential sources of metabolic drug interactions. Toxicology 2010;270:59-65.

24. Fleming $C D$, Bencharit S, Edwards CC, et al. Structural insights into drug processing by human carboxylesterase 1 : tamoxifen, mevastatin, and inhibition by benzil. J Mol Biol 2005;352:165-77.

25. Fukami T, Takahashi S, Nakagawa N, Maruichi T, Nakajima M, Yokoi T. In vitro evaluation of inhibitory effects of antidiabetic and antihyperlipidemic drugs on human carboxylesterase activities. Drug Metab Dispos 2010;38:2173-8.

26. Zhang J, Burnell JC, Dumaual N, Bosron WF. Binding and hydrolysis of meperidine by human liver carboxylesterase hCE-1. J Pharmacol Exp Ther 1999;290:314-38.

27. Holodniy M, Penzak SR, Straight TM, et al. Pharmacokinetics and tolerability of oseltamivir combined with probenecid. Antimicrob Agents Chemother 2008;52:3013-21.

28. Schentag JJ, Hill G, Chu T, Rayner CR. Similarity in pharmacokinetics of oseltamivir and oseltamivir carboxylate in Japanese and Caucasian subjects. J Clin Pharmacol 2007;47:68996.

29. Wildschut ED, de Hoog M, Ahsman MJ, Tibboel D, Osterhaus AD, Fraaij PL. Plasma concentrations of oseltamivir and oseltamivir carboxylate in critically ill children on extracorporeal membrane oxygenation support. PLoS One 2010;5: el0938.

30. Lemaitre F, Luyt CE, Roullet-Renoleau F, et al. Oseltamivir carboxylate accumulation in a patient treated by haemodiafiltration and extracorporeal membrane oxygenation. Intensive Care Med 2010;36:1273-4.

31. Gubavera L, Okomo-Adhiambo M, Deyde V, et al. Update: drug susceptibility of swine-origin influenza A ( $\mathrm{H} 1 \mathrm{Nl})$ viruses, April 2009. MMWR 2009;58:433-5.

32. Wattanagoon $\mathrm{Y}$, Stepniewska $\mathrm{K}$, Lindegårdh $\mathrm{N}$, et al. Pharmacokinetics of high-dose oseltamivir in healthy volunteers. Antimicrob Agents Chemother 2009;53:945-52. 